\title{
No evidence for pathogenic variants or maternal effect of ZFP57 as the cause of Beckwith-Wiedemann Syndrome
}

\author{
Susanne E Boonen ${ }^{\star, 1,2}$, Johanne MD Hahnemann ${ }^{1}$, Deborah Mackay ${ }^{3,4}$, Niels Tommerup ${ }^{2}$, \\ Karen Brøndum-Nielsen ${ }^{1,5}$, Zeynep Tümer ${ }^{1,5}$ and Karen Grønskov ${ }^{1,5}$
}

Beckwith-Wiedemann syndrome (BWS) is an overgrowth syndrome, which, in $50-60 \%$ of sporadic cases, is caused by hypomethylation of KCNQ1OT1 differentially methylated region (DMR) at chromosome 11p15.5. The underlying defect of this hypomethylation is largely unknown. Recently, recessive mutations of the ZFP57 gene were reported in patients with transient neonatal diabetes mellitus type 1, showing hypomethylation at multiple imprinted loci, including KCNQ1OT1 DMR in some. The aim of our study was to determine whether ZFP57 alterations were a genetic cause of the hypomethylation at KCNQ1OT1 DMR in patients with BWS. We sequenced ZFP57 in 27 BWS probands and in 23 available mothers to test for a maternal effect. We identified three novel, presumably benign sequence variants in ZFP57; thus, we found no evidence for ZFP57 alterations as a major cause in sporadic BWS cases.

European Journal of Human Genetics (2012) 20, 119-121; doi:10.1038/ejhg.2011.140; published online 24 August 2011

Keywords: Beckwith-Wiedemann syndrome; imprinting; hypomethylation; ZFP57; maternal effect genes; germline alterations

\section{INTRODUCTION}

Beckwith-Wiedemann syndrome (BWS, OMIM 130650) is an imprinting syndrome with an incidence of $1: 13700{ }^{1}$ The most prominent clinical features are pre- and postnatal overgrowth ( $>97$ th centile), macroglossia and abdominal wall defects. ${ }^{1-3}$ The underlying pathology of BWS includes genetic and epigenetic alterations on chromosome 11p15.5, resulting in dysregulation of growth regulatory genes. ${ }^{1-3}$ Hypomethylation of the differentially methylated region (DMR) of imprinting control region 2/KCNQ1OT1 DMR on the maternal allele occurs in $50-60 \%$ of BWS cases and is mostly due to an apparently pure epigenetic modification. ${ }^{1,2}$

Recently, mechanisms influencing imprinting disorders in trans have been identified. Hypomethylation of multiple imprinted loci (HIL) is described in different imprinting syndromes including transient neonatal diabetes mellitus type 1 (TNDM1; MIM 601410), ${ }^{4}$ BWS, ${ }^{5-7}$ Silver-Russell syndrome (SRS; MIM 180860)/growth restriction, ${ }^{8,9}$ and in a single patient with the clinical phenotype of BWS and Prader-Willi syndrome. ${ }^{10}$ Recessive ZFP57 mutations were identified in more than half of the TNDM1 cases displaying HIL. ${ }^{11}$ ZFP57 is localized at chromosome $6 \mathrm{p} 22.1$ and encodes the KRAB zinc finger protein ZFP57, containing seven zinc finger domains (ZF1-ZF7). ${ }^{11} \mathrm{In}$ mice, when the zygotic function of $Z f p 57$ is lost $\left(Z f p 57^{-/-}\right)$, offsprings show partial hypomethylation at multiple maternally and paternally imprinted loci, but when both maternal and zygotic zfp57 function are lost $\left(Z f p 57^{-1-}\right.$ offspring of $Z f p 57^{-1-}$ mothers), the offsprings show complete loss of methylation. This indicates that $Z f p 57$ is a maternalzygotic effect gene required for maintenance of DNA methylation imprints. ${ }^{12,13}$ The maternal effect of a gene is the genetic phenomenon in which a phenotype in the progeny is caused by a genetic alteration in the maternal genome rather than an alteration of its own; this has been observed both in humans ${ }^{7,14}$ and in mice. ${ }^{12,13}$ Mutations in NLRP7 were described in females with familial recurrent biparental complete hydatidiform moles. ${ }^{14}$ These females had normal methylation, but failed to establish methylation imprints in their oocytes. More recently, a homozygous NLRP2 mutation was found in a female; both her sons had BWS with hypomethylation of the KCNQ1OT1 DMR, and furthermore, one of them also had hypomethylation of PEG1 DMR. ${ }^{7}$ In this study, we present the mutation analysis of ZFP57 in sporadic BWS patients and their mothers to test two hypotheses: (1) mutations in ZFP57 lead to isolated hypomethylation of KCNQ1OT1 DMR and thereby lead to BWS; (2) mutations in ZFP57 in a mother may lead to BWS in her children.

\section{MATERIAL AND METHODS}

Study population

Twenty-seven sporadic BWS probands (14 females and 13 males), 23 from Scandinavia, three from other parts of Èurope and one of unknown origin, were referred for diagnostic molecular genetic testing for BWS. Patients with hypomethylation at KCNQ1OT1 DMR were selected for the study. This was tested either by Southern blot analysis using a KCNQ1OT1 probe and a methylation sensitive restriction enzyme, or by methylation-specific MLPA (MS-MLPA) analysis using kit ME030 (MRC-Holland, Amsterdam, the Netherlands). The mean methylation indices were for Southern blot analysis 0.12 (based on nine patients; minimum 0.05 and maximum 0.24; median 0.07) and for MS-MLPA 0.09 (based on four patients; minimum 0.01

${ }^{1}$ Center for Applied Human Molecular Genetics, The Kennedy Center, Glostrup, Denmark; ${ }^{2}$ Wilhelm Johannsen Centre for Functional Genome Research, Department of Cellular and Molecular Medicine, Panum Institute, University of Copenhagen, Copenhagen N, Denmark; ${ }^{3}$ Wessex Genetics Service, Southampton University Hospital Trusts, Southampton SO16 5Y, UK; ${ }^{4}$ Salisbury Hospital NHS Foundation Trust, Salisbury, UK; ${ }^{5}$ Faculty of Health Sciences, University of Copenhagen, Copenhagen, Denmark

*Correspondence: Dr SE Boonen, Center for Applied Human Molecular Genetics, The Kennedy Center, GI. Landevej 7, 2600 Glostrup, Denmark. Tel: +45 43260168

Fax: + 45434311 30; E-mail: seb@kennedy.dk

Received 25 January 2011; revised 29 June 2011; accepted 30 June 2011; published online 24 August 2011 
and maximum 0.14; median 0.10). Ten patients were further tested for HIL by MS-PCR as described previously. ${ }^{4}$ Three of the 27 probands were from discordant twin pairs (one from a pair of female monozygotic (MZ) twins, one from a pair of female twins with unknown zygosity, and one from a pair of male twins of unknown zygosity). Twenty-three mothers of BWS probands were available for investigation, and all had normal methylation at KCNQ1OT1 DMR tested as described above. To our knowledge, none of the probands were from consanguineous families. We have no information about whether the probands were conceived by assisted reproductive technology. The control group consisted of 50 normal Danish individuals.

\section{DNA studies}

Mutation analysis. DNA was extracted from peripheral blood by standard procedures. The five coding exons of ZFP57 and $20 \mathrm{bp}$ of the flanking intronic sequences were amplified in eight fragments by PCR as previously described. ${ }^{11}$ PCR primer sequenced are listed in Supplementary Table 1 and PCR conditions are available upon request. All ZFP57 alterations are described according to den Dunnen and Antonarakis, ${ }^{15}$ with GenBank accession number: NM_001109809.1.

\section{In silico analysis}

The possible impact of the novel amino acid substitutions was evaluated using the SIFT database, (http://sift.jcvi.org/) and the PolyPhen-2 database, (http://genetics.bwh.harvard.edu/pph2/).

\section{RESULTS}

By sequencing ZFP57 in 27 sporadic BWS patients with hypomethylation at KCNQ1OT1 DMR and in 23 mothers with normal methylation, we identified three novel sequence variations. All the variations were located in exon 6 . Ten out of the 27 patients were investigated for HIL, but no signs of HIL were observed (data not shown).

In the affected twin from the MZ female twins (from Scandinavia), we detected a heterozygous sequence variation c.503C $>\mathrm{T}$ (p.Ser168Phe) affecting a non-conserved amino acid in ZF1. The variation was also found in the non-affected twin and in the father, but not in the mother. The SIFT database predicts the variation to be tolerated with a score of 0.30 (normal >0.05), but the PolyPhen-2 database predicts it to be probably damaging with a score of 0.971 (sensitivity: 0.56 ; specificity: 0.91 ). The variant was not detected in 50 controls or present in the 1000 genomes database (http://browser. 1000genomes.org/index.html).

In a proband from Europe, we found two silent sequence variations, $\mathrm{c} .723 \mathrm{C}>\mathrm{T}(\mathrm{p} .=)$ and $\mathrm{c} .1026 \mathrm{~T}>\mathrm{C}(\mathrm{p} .=)$. The c.723C $>\mathrm{T}$ is located in ZF4 and the c.1026T $>C$ is located between ZF4 and ZF5. Both variations were inherited from the unaffected father and thus were in cis. Both variations were absent in the mother. The variants were not detected in 50 controls or in the 1000 genome database.

In a proband from Scandinavia, we identified a sequence variation c.499C $>$ T (p.Arg167Cys) affecting a non-conserved amino acid in $\mathrm{ZF1}$. The variation was inherited from the mother. This variation was present in seven out of 50 controls and was reported in the SNP database (http://ncbi.nlm.nih.gov/snp) (rs61730330). The SIFT database predicts the variation to affect protein function with a score of 0.02 (normal $>0.05$ ) and the PolyPhen-2 database predicts it to be probably damaging with a score of 0.914 (sensitivity: 0.67; specificity: 0.86).

\section{DISCUSSION}

The etiology of the hypomethylation of KCNQ1OT1 DMR observed in BWS patients is unknown. Recently, mutations in ZFP57 were found to cause HIL in some TNDM1 patients. ${ }^{11}$ As KCNQ1OT1 DMR is among the loci observed to be hypomethylated in TNDM HIL patients, and further, as some BWS patients were also shown to have
HIL, we investigated whether sporadic BWS could be caused by mutations in ZFP57.

We identified three novel sequence variations in ZFP57. Two of the variations are silent mutations, and furthermore, not located at the splice sites. The causative effect of the third variation is questionable as suggested by in silico analysis. We also identified a known SNP. None of the probands or mothers was homozygous or compound heterozygous for variations in ZFP57.

It could be argued that ZFP57 mutations would only be found in patients with HIL, but notably the methylation profile for the different imprinted loci varies considerably in patients with ZFP57 mutations. ${ }^{11}$ Other studies reported $11-25 \%$ of BWS patients ${ }^{5,6}$ and $14 \%$ of SRS patients ${ }^{8,9}$ to have HIL. In our BWS cohort, none of the 10 investigated patients had HIL, but the cohort is too small to deduce conclusions. In SRS patients, mutational analysis of ZFP57 similarly has shown no pathogenic alterations. ${ }^{16}$ In contrast to BWS, however, the hypomethylation in SRS affects a paternally imprinted locus (H19 DMR), albeit cases of both SRS and BWS have been reported with hypomethylation at both maternally as well as paternally methylated DMRs. ${ }^{8,9,17,18}$

Other trans-acting factors and mechanisms involved in the establishment and maintenance of methylation in DNA imprints could be responsible for the methylation and demethylation at KCNQ1OT1, maybe in cooperation with ZFP57, including DNA methyltransferases, methyl-binding proteins, imprinting genes network and/or RNA interference. Furthermore, alterations in cis are known to cause aberrant methylation, for example, in SRS and growth retardation where deletions and translocations of the H19/IGF2 enhancer region can cause hypomethylation of the IGF2P0 promoter element. ${ }^{19}$

Mice studies have shown that $Z f p 57$ is a maternal effect gene, and it has been hypothesized that a gradual loss of DNA methylation takes place in the Zfp57-I- progeny of Zfp57-I- mothers and account for the spatial and temporal discrepancy of the molecular defect and the phenotypic effect; ${ }^{13}$ however, we do not find ZFP57 to be a major maternal effect gene in sporadic BWS, neither do our data support the hypothesis that alterations in ZFP57 are a major cause of KCNQ1OT1 DMR hypomethylation in sporadic BWS cases.

\section{CONFLICT OF INTEREST}

The authors declare no conflict of interest.

\section{ACKNOWLEDGEMENTS}

Wilhelm Johannsen Centre for Functional Genome Research is established by the Danish National Research Foundation. This work was supported by a grant from the Danish Agency for Science, Technology and Innovation, the University of Copenhagen. Furthermore, financial support was granted by Director Jacob Madsen and wife Olga Madsens Foundation and King Christian X fond.

1 Weksberg R, Shuman C, Beckwith JB: Beckwith-Wiedemann syndrome. Eur J Hum Genet 2010; 18: 8-14.

2 Choufani S, Shuman C, Weksberg R: Beckwith-Wiedemann syndrome. Am J Med Genet C Semin Med Genet 154C 2010; 3: 343-354.

3 Weksberg R, Shuman C, Smith AC: Beckwith-Wiedemann syndrome. Am J Med Genet C Semin Med Genet 137C 2005; 1: 12-23.

4 Mackay DJ, Hahnemann JM, Boonen SE et al: Epimutation of the TNDM locus and the Beckwith-Wiedemann syndrome centromeric locus in individuals with transient neonatal diabetes mellitus. Hum Genet 2006; 119: 179-184.

5 Bliek J, Verde G, Callaway J et al: Hypomethylation at multiple maternally methylated imprinted regions including PLAGL1 and GNAS loci in Beckwith-Wiedemann syndrome. Eur J Hum Genet 2009; 17: 611-619. 
6 Rossignol S, Steunou V, Chalas C et al: The epigenetic imprinting defect of patients with Beckwith-Wiedemann syndrome born after assisted reproductive technology is not restricted to the 11 p15 region. J Med Genet 2006; 43: 902-907.

7 Meyer E, Lim D, Pasha S et al: Germline mutation in NLRP2 (NALP2) in a familial imprinting disorder (Beckwith-Wiedemann Syndrome). PLoS Genet 2009; 5: e1000423.

8 Azzi S, Rossignol S, Steunou V et al: Multilocus methylation analysis in a large cohort of 11p15-related foetal growth disorders (Russell Silver and Beckwith Wiedemann syndromes) reveals simultaneous loss of methylation at paternal and maternal imprinted loci. Hum Mol Genet 2009; 18: 4724-4733.

9 Turner CL, Mackay DM, Callaway JL et al: Methylation analysis of 79 patients with growth restriction reveals novel patterns of methylation change at imprinted loci. Eur J Hum Genet 2010; 18: 648-655.

10 Baple EL, Poole RL, Mansour S et al: An atypical case of hypomethylation at multiple imprinted loci. Eur J Hum Genet 2011; 19: 360-362.

11 Mackay DJ, Callaway JL, Marks SM et al: Hypomethylation of multiple imprinted loci in individuals with transient neonatal diabetes is associated with mutations in ZFP57. Nat Genet 2008; 40: 949-951.

$12 \mathrm{Li} \mathrm{X}$, Ito M, Zhou $\mathrm{F}$ et al: A maternal-zygotic effect gene, Zfp57, maintains both maternal and paternal imprints. Dev Cell 2008; 15: 547-557.
13 Li X: Extending the maternal-zygotic effect with genomic imprinting. Mol Hum Reprod 2010; 16: 695-703.

14 Murdoch S, Djuric U, Mazhar B et al: Mutations in NALP7 cause recurrent hydatidiform moles and reproductive wastage in humans. Nat Genet 2006; 38 : 300-302.

15 den Dunnen JT, Antonarakis SE: Mutation nomenclature extensions and suggestions to describe complex mutations: a discussion. Hum Mutat 2000; 15: 7-12.

16 Spengler S, Gogiel M, Schonherr N, Binder G, Eggermann T: Screening for genomic variants in ZFP57 in Silver-Russell syndrome patients with 11 p15 epimutations. Eur J Med Genet 2009; 52: 415-416.

17 Begemann M, Spengler S, Kanber D et al: Silver-Russell patients showing a broad range of ICR1 and ICR2 hypomethylation in different tissues. Clin Genet 2011; 80: 83-88.

18 Bliek J, Alders M, Maas SM et al: Lessons from BWS twins: complex maternal and paternal hypomethylation and a common source of haematopoietic stem cells. Eur J Hum Genet 2009; 17: 1625-1634.

19 Gronskov K, Poole RL, Hahnemann JM et al: Deletions and rearrangements of the $\mathrm{H} 19$ / IGF2 enhancer region in patients with Silver-Russell syndrome and growth retardation. J Med Genet 2011; 48: 308-311.

Supplementary Information accompanies the paper on European Journal of Human Genetics website (http://www.nature.com/ejhg) 